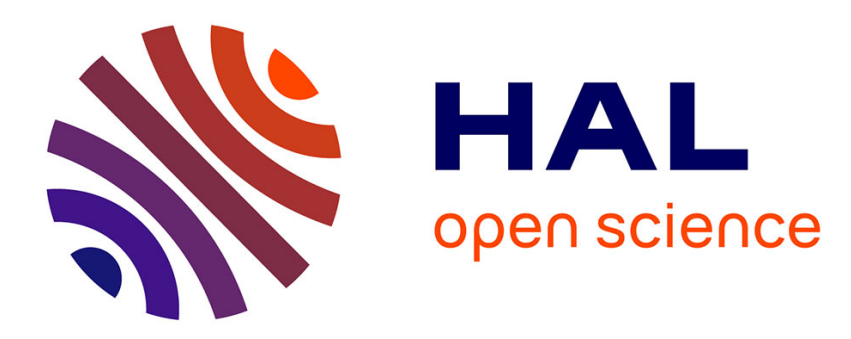

\title{
The Limits of Molecular Dynamics Applied to Condensed-Phase Energetic Materials
}

\author{
E. Oran, J. Boris
}

\section{To cite this version:}

E. Oran, J. Boris. The Limits of Molecular Dynamics Applied to Condensed-Phase Energetic Materials. Journal de Physique IV Proceedings, 1995, 05 (C4), pp.C4-609-C4-617. 10.1051/jp4:1995450 . jpa-00253756

\section{HAL Id: jpa-00253756 https://hal.science/jpa-00253756}

Submitted on 1 Jan 1995

HAL is a multi-disciplinary open access archive for the deposit and dissemination of scientific research documents, whether they are published or not. The documents may come from teaching and research institutions in France or abroad, or from public or private research centers.
L'archive ouverte pluridisciplinaire HAL, est destinée au dépôt et à la diffusion de documents scientifiques de niveau recherche, publiés ou non, émanant des établissements d'enseignement et de recherche français ou étrangers, des laboratoires publics ou privés. 


\title{
The Limits of Molecular Dynamics Applied to Condensed-Phase Energetic Materials
}

\author{
E.S. Oran and J.P. Boris \\ Laboratory for Computational Physics, Naval Research Laboratory, Washington, DC 20376, U.S.A.
}

\begin{abstract}
The limitations of various methods for computing manybody dynamics are summarized briefly in terms of the physical limits of the specific theory and generally of what can reasonably be computed. This information is then used to assess the current computational limit on using molecular dynamics to describe shocks and detonations in condensed phase energetic materials. This question is addressed by defining the computational requirements of a molecular dynamics simulation of a detonation propagating in an idealized nitromethane crystal lattice. The major questions addressed are: What is required to compute the properties of the system to obtain reasonable mesoscopic data? and What is the size of the system we can now compute, using one of the largest computers available? From this analysis, we discuss several directions in which future research in this field may proceed.
\end{abstract}

\section{INTRODUCTION}

In a manybody-dynamics calculation, a large number of individual particles represent entities such as atoms, molecules, droplets, clusters, stars, or galaxies. These particles move according to prescribed equations of motion and interact according to specified force laws. In principle, the equations of motion can have any form. They could, for example, be classical, quantum mechanical, or relativistic. In the same sense, the interactions among the particles could be, for example, hard-sphere, two-body, manybody, gravitational, or electromagnetic. Once the particles and their interactions are defined, the system is allowed to evolve according to these laws. Applications of manybody dynamics have ranged from studies of phase transitions in liquids and solids, dynamics of large molecules, materials processing in dense systems, and galaxies forming from star clusters.

Several aspects of molecular dynamics are important for making decisions about how and and under what conditions the method can be applied. Molecular dynamics is deterministic, in contrast to any form of Monte Carlo method, in which some elements of a statistical nature are introduced. Molecular dynamics is computer intensive. Computing the behavior of real physical systems, with realistic sizes and interaction, requires a great deal of computer time and memory. Molecular dynamics requires attention to selecting the best algorithms for tracking and evaluating the interactions among large number of particles. Major decisions in setting up the physics 
in molecular-dynamics simulations involve defining the nature of the interacting particles and their interactions, given the current knowledge of the interaction and the computational resources available. Usually tradeoffs between accuracy, computational efficiency, and realistic physical complexity must be made to obtain any reasonable results.

The objective of this paper is to clarify the limits, both physical and computational, of using molecular dynamics to describe shocks and detonations in condensed phase energetic materials. This perspective should help determine which directions might profitably be followed in the near future. To help understand these limits, it is first necessary to evaluate the inherent limits of various approaches and therefore how molecular dynamics fits into the scheme of theories for solving manybody interactions. Since molecular dynamics relies on the availability of computational power, we consider what computers can now accomplish, and what they are likely to achieve in the near future. Finally, we use this information to evaluate what can be done in the study of ignition and propagation of shock and detonation waves in energetic materials.

\section{APPROACHES TO SOLVING MANYBODY INTERACTIONS}

Table 1 shows the heirarchy of mathematical models used to describe the behavior of systems involving many particles and interactions. Molecular dynamics is the most fundamental level of this heirarchy, and the interactions may be as basic as Newton's Law. Both Monte Carlo approaches and the Boltzmann equation are derived from the Liouville equation. At the level where particles can be considered a continuum fluid, the Navier-Stokes equations are generally used.

Some of the information in Table 1 is recast into quantitative form in Figure 1, which shows the physical regimes of validity of the methods (modified from Bird [1]). The regimes of validity are expressed as a function of a characteristic distance in the system $L$, and the mean molecular spacing, $\delta$. For a fixed molecular diameter, $d$, as the mean spacing between molecules decreases, the density increases. This is shown on the horizontal axis. The figure shows limits of the NavierStokes, DSMC, and molecular dynamics models, methods that can, in principle, be used to describe real physical systems.

Table 1. Levels of Models of Manybody Interactions

Equation Solution Method

Newton's Laws

$$
f=m a
$$

Liouville Equation

$$
F\left(\mathbf{x}_{i}, \mathbf{v}_{i}, t\right), \quad i=1, N_{p}
$$

Boltzmann Equation

$F(\mathbf{x}, \mathbf{v}, t)$

binary collisions (low density)

good for gases

Navier-Stokes Equation

$\rho(\mathbf{x}, t), \quad \mathbf{u}(\mathbf{x}, t)$

short mean free path (small $K n$ )
Molecular Dynamics

Monte Carlo Methods

Direct Simulation Monte Carlo

Direct Solution

Direct Solution: Finite Differences, Finite Volumes, Spectral Methods ... 


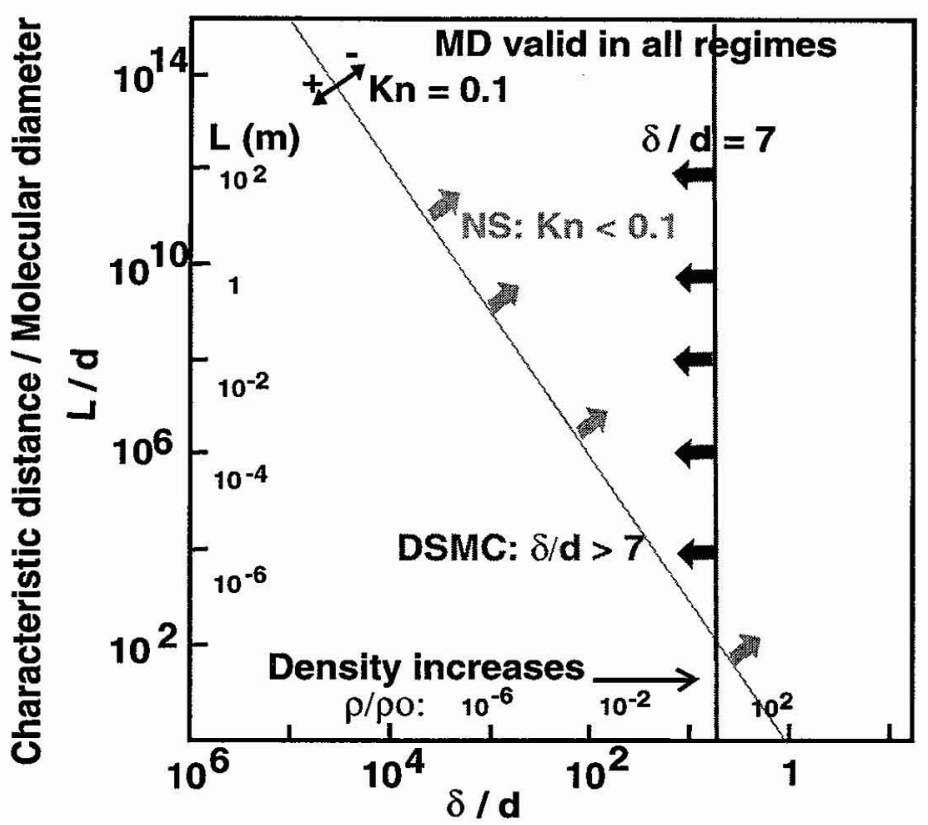

Mean molecular spacing / Molecular diameter

Figure 1. Regimes of validity of molecular dynamics, Direct Simulation Monte Carlo, and Navier Stokes, as a function of the characteristic length scale and mean molecular spacing of a system.

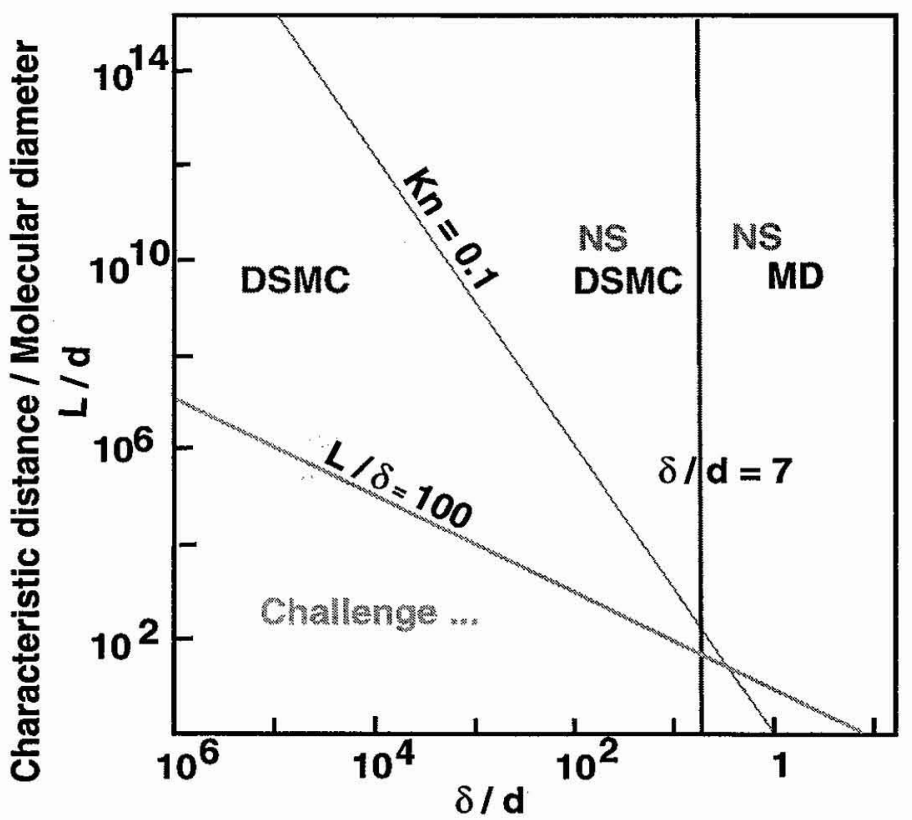

Mean molecular spacing / Molecular diameter

Figure 2. Regimes where various methods can be used, given current computers. 
The Navier-Stokes equations are generally applicable when the system behaves as a continuum fluid. This is the case when the Knudsen number, the ratio of the molecular mean free path to a characteristic system length, is small,

$$
K_{n} \equiv \lambda / L<0.1 \text {. }
$$

The Navier-Stokes equations could be modified to describe flows at higher Knudsen numbers, but to do this it is necessary to reformulate the expressions for heat and mass diffusion. Efforts to do this have been successful in slightly extending the range of validity. Efforts to use continuum approximations for higher Kundsen number situations involve solving equation sets that are mathematically of higher order than the Navier-Stokes equations. Examples of these include the Burnett equations and various moment methods, which are computationally more expensive, more difficult to implement, and less well calibrated than the Navier-Stokes equations.

When the ratio of the mean molecular spacing, $\delta$, to the molecular diameter, $d$, is large, so that $\delta / d>>1$, the Direct Simulation Monte Carlo method can be used. It is reasonable to take $\delta / d>7[1]$. The DSMC method is statistically based, and generally applied to high-Knudsennumber flows. It has been used extensively for atmospheric reentry problems, where the gas is very dilute, and now it is being used for denser flows in microchannels. The method is based on the same basic approximations from which the Boltzmann equation is derived, but its regime of validity also extends to systems where three-body collisions can occur. Because of the different criteria for validity of Navier-Stokes and DSMC, there is a range in which both methods can be used. Both methods are valid in the triangular domain of Figure 1 where $\delta / d>7$ but $K n<0.1$.

Molecular dynamics is valid throughout the entire range of parameters. This is because it is based on the most fundamental set of equations. There are no physical reasons why it cannot be used for all ranges of densities and system sizes.

\section{THE COMPUTATIONAL LIMITS}

The boundary lines between various methods shown in Figure 1 are based on very general physical principles, unrelated to either the limitations of input data or computational capability. Figure 2 is an attempt to further put limits on the applicability of a method by looking at the computational limits.

Navier-Stokes methods are valid for low Knudsen numbers at high enough densities. When the Knudsen number is close to 0.1 , correction terms are necessary. The DSMC methods can be used for high Knudsen number fluids, and again it is still possible to use them for the regimes of overlap with Navier-Stokes, where $K_{n}<0.1$ and $\delta / d>7$, but here the computations become quite expensive.

Molecular dynamics, however, becomes expensive for low density problems. For this method to be useful, we need to compute long enough for the system to evolve on the macroscopic time and space scales while using a specified microscopic particle interaction law. The computational timestep, however, is limited by the timescale of the interaction potential and the method of solution. If the particles are too far apart, it could take thousands or even millions of timesteps to simulate a few collisions. The expense becomes exorbitant. Thus molecular dynamics can be too expensive for dilute systems. This prohibition also extends to very large systems and systems that evolve slowly.

The regime of parameter space in which $L / \delta<100$ is one in which there are few particles per volume element, and therefore few collisions. When this is the case, there are large fluctuations in the calculations of the mean properties of the system, such as density, pressure, and temperature. A DSMC calculation would require many ensembles to get good statistics. A molecular dynamics calculation would produce large spatial fluctuations in mean quantities. A Navier-Stokes computation would not necessarily show these physically realistic fluctuations, which would have to be 
added by other theories. One extreme is the free-molecule limit, where a collisionless Boltzmann equation applies.

\section{MOLECULAR DYNAMICS AND THE STUDY OF ENERGETIC MATERIALS}

Substantial efforts have gone into using molecular dynamics to study the behavior of shocks and detonations in condensed phase energetic materials. The pioneering work of Karo and Walker [2], Odiot et al. [3-5], and Tsai and Trevino [6] inspired the later work by authors such as Lambrakos et al. [7], White and Brennan [8], and Phillips et al. [9]. All of these molecular dynamics computations used model systems that attempted to incorporate some of the important characteristics of condensed phase energetic materials. In some cases, the systems were treated as two dimensional. Simplified force laws are used, and complex energetic molecules are generally approximated by combining a number of atoms into a smaller number of macroparticles.

These idealized and scaled studies have produced a number of interesting results. The simulations showed that an ordered lattice structure, as in a solid, or a group of more-randomly-oriented dense particles, as in a liquid, can sustain a wave that behaves like a shock wave. Further, if there is energy added close behind the shock wave, the result is a propagating wave structure that looks like a detonation. As in the continuum representation of a detonation, the energy addition contributes to the molecular motion and drives the shock even faster. These results answered some of the first questions about molecular dynamics that were asked by the detonation-physics community: Could a system of particles, interacting in prescribed ways, mimic the understood behavior of a detonation? Once this was shown, it was then possible to ask questions such as how does a supersonic wave pass through a lattice, and what does it do to the lattice as it propagates? Is there a minimum ignition energy required to initiate a detonation? How can you ignite a detonation? What are the differences between a detonation propagating in a lattice and in a liquid? What are the differences between one-dimensional, two-dimensional, and three-dimensional representations of a system? What are the effects of vacancies and mass defects? All of these and other questions have been addressed. Taken together, the simulations performed to answer these questions have given a basic understanding of how a lattice reacts as it is perturbed by highly nonlinear forces and interactions.

\section{THE LIMITS OF THESE STUDIES}

In the computations discussed above, the system parameters had to be scaled to carry out the simulations. With the resources available, we could not consider simulating detonation ignition or propagation in systems with realistic values of the system size, $L$, and rates of energy release. To decrease the size of the system and have a viable simulation, the rates at which chemical reactions occur and energy is released must be increased. These types of scaled, approximate models have been used for decades in plasma simulations where much work has been done for proton to electron mass ratios much smaller than the correct value of 1836 . This scaling is done with the hope that the same types of effects that occur in actual systems can be studied on a smaller, faster scale. Experimental studies have shown that the sizes and times of the actual effects simulated are orders of magnitude larger than the ones simulated. In such small systems, we can see some of the basic features of wave propagation, but we cannot simultaneously resolve the macroscale processes that we know are important. For example, we can only barely begin to see the effects of coherent waves structures and grain interactions. The question we ask is whether molecular dynamics can be used to compute the kinds of properties of energetic materials that can be compared directly to experimental results? Will we always be confined to "model" systems and scaled parameters?

Consider the more specific question: How much computational power is needed to model a microscopic system with enough particles to constitute a sample large enough to see macroscopic 
effects? For example, with the largest new computers, we can consider simulating the behavior of a billion individual particles and simultaneously computing their interactions at rates of tens of gigaflops. (One gigaflop is a billion floating point operations per second.) Would a billion particles be enough?

To help address this question, we focus on a specific type of computation that has been optimized on the largest computers: a model system of a nitromethane crystal. The system could also be a liquid, and the same estimates would hold.) We also consider a detonation propagation problem. An ignition problem would require simulating a much larger systems for longer physical times. We assume that the bulk of the energy is released from a shocked element of nitromethane in $5 \mu \mathrm{m}$, an estimate that is perhaps too small by a factor of five. An earlier Navier-Stokes computations, describing detonation cells in liquid nitromethane, showed that the detonation cell height is $50 \mu \mathrm{m}$, and so the detonation cell length is less than $100 \mu \mathrm{m}$.

Therefore, a minimum macroscopic scale (a mesoscale), is at least a couple of detonation cell lengths long, and at least one cell high. The detonation would have to travel further than this in the calculation, but this can be achieved numerically by adding unreacted material ahead of the detonation, and subtracting it from the back. To look at macroscopic phenomena, however, at least one or two cell lengths should be retained in the computer at any on time. Thus our assumption here is that we need to simulate a system that is $100 \mu \mathrm{m} \times 50 \mu \mathrm{m}$ in two dimensions, and $100 \mu \mathrm{m}$ $\times 50 \mu \mathrm{m} \times 50 \mu \mathrm{m}$ in three dimensions. We also assume that the detonation travels at about $5000 \mathrm{~m} / \mathrm{s}$. Therefore, for the detonation to propagate $100 \mu \mathrm{m}$ requires that we simulate $2 \times 10^{-8} \mathrm{~s}$ of physical time. For a typical computational time step of $10^{-13} \mathrm{~s}$ (perhaps too long for realistic simulations), we need to be able to compute $2 \times 10^{5}$ time steps for the detonation to propagate approximately one detonation cell length. A usual computation would require computing for a number of cell lengths, say four or five, or about $10^{6}$ timesteps.

Let us consider the simplest model, the $\mathrm{C}-\mathrm{N}$ model, in which the $\mathrm{C}$ represents $\mathrm{CH}_{3}$, and the $\mathrm{N}$ represents $\mathrm{NO}_{2}[4,7]$. The initial shock can create conditions in which the system is electronically excited, and the C-N bond can break. After it has broken, other reactions take place which eventually lead to the formation of final products and energy release. This second process is modeled by a time delay to energy release after the C-N breakup. This extremely simple model contains the essence of what is needed to generate a reaction wave in a system. If the energy release occurs close enough behind the leading shock, it can couple to it dynamically and evolve into a detonation. If the energy release it too slow, a slower deflagration or flame may arise.

Molecular dynamics studies have shown that each pair of C-N's are separated by about 5 A. In two-dimensional studies, the C-N's can form a minimum-energy lattice by aligning with all of the C-N's point in the same direction. In three-dimensions, there are two minimum energy states. In the lowest, the unit cell is $10 \mathrm{~A}$, and the C-N's are aligned in opposite directions every $5 \mathrm{~A}$. In the metastable state, the unit cell is about approximately $5 \mathrm{~A}$, and the C-N's are aligned. We also assume that the relatively simple form of a potential, such as the predissociative potentials [4], are used in the hypothetical calculation.

Recent computations have shown that there are substantial differences between the results of two- and three-dimensional computations [9]. The additional degrees of freedom in three dimensions means that the system can equilibrate more quickly than it would in two dimensions, and so the time scales change. Therefore, anomalous effects occur in simulations in two dimensions, and these effects disappear in three dimensions. For example, in two dimensions, there is a sharp transition from an initial detonation state that depends on initial input energy to one that correspond to the Chapman-Jouguet state. Computations in three dimensions go smoothly to this final state. Our conclusion is that to obtain quantitatively valid simulations involving flows of material in transition, it is necessary to perform three-dimensional calculations. For these particular systems, simulating three-dimensional situations using molecular dynamics may be more important than it 
is using a fluid dynamics approach. In fluid dynamics, the equations of state already includes the microscopic effects of three dimensions, even in two-dimensional simulations.

Combining this information about the model lattice and what constitutes a minimum system size to obtain a meaningful mesoscopic simulation, allows us to compute the number for particles that must be included in such a simulation. The result is that a two-dimensional simulation needs at least $4 \times 10^{11}$ particles, and a three-dimensional simulation should have at least $8 \times 10^{16}$ particles. This is considerably larger than the billion particles that we can now contemplate simulating and the 600 million particles treated in some simulations to date. These estimates are summarized in Table 2.

For a two-dimensional molecular dynamics simulation using the model given in Table 2, we need approximately 10 arrays. For a three-dimensional calculation, we need to store approximately 12 arrays. These numbers are not exact, because it is often possible to trade off memory storage for computer time. Some variables, that for convenience could be stored in computer memory, can be recomputed from other stored variables. Also, depending on whether the computation is done in single or double precision, approximately $40-80$ bytes of storage per particle is required in two dimensions and 50-100 bytes/particle in three dimensions. The result is that 8 terabytes of memory are needed for a two-dimensional calculation, and 4 million terabytes for a threedimensional computation.

At this point there is a natural question to ask: What we can simulate now? First, consider what is actually available in terms of computer resources. These estimates are for the CM-5E at NRL, a massively parallel supercomputer on which most of our molecular dynamics codes are optimized. The CM has 32 Gbytes of memory. Therefore, we definitely do not have the computational memory available to carry out even the $4 \times 10^{11}$ particle simulation. The CM can reach a theoretical speed of 40 Gflops, and practical programs can achieve about 8 Gflops. This is painfully inadequate. The computation of one time step requires about 500 operations/particle/timestep in two dimensions, and 1000 operations/particle/timestep in three dimensions. Therefore, at a speed of 8 Gflops, one time step of a two-dimensional calculation would take 7 hours to compute, and one timestep of a three-dimensional calculation would take 300 years! We are hardly able to compute for one time step, let alone the $10^{5}$ or $10^{6}$ timesteps it would take for the detonation to propagate a cell length.

Table 2. Summary of Physical Model and Computational Requirements

Physical Model Modified C-N predissociative model for crystal nitromethane.

Intramolecular spacing $5 \mathrm{~A}$.

Detonation speed $5000 \mathrm{~m} / \mathrm{s}$.

Minimum Macroscopic Scale

2D $\quad 100 \times 50 \mu \mathrm{m}^{2}$

3D $100 \times 50 \times 50 \mathrm{~mm}^{3}$

Number of Simulated Particles

2D $4 \times 10^{11}$

3D $8 \times 10^{16}$

Computational Storage Requirements:

2D 40-80 bytes/particle Total 8 Tbytes

3D 50-100 bytes/particle Total $4 \times 10^{6}$ Tbytes 
What is close to computable is a system that is $0.2 \mu \mathrm{m} \times 0.2 \mu \mathrm{m} \times 0.4 \mu \mathrm{m}$, which, for this physical model, would require $1.6 \times 10^{7}$ particles, or perhaps $0.5 \mu \mathrm{m} \times 0.5 \mu \mathrm{m} \times 1.0 \mu \mathrm{m}$, which would require $2.5 \times 10^{8}$ particles. This is about the limit that will fit into a full 32 Gbytes. This larger system would require integrating $2.0 \times 10^{-10} \mathrm{~s}$ of physical time for a detonation cell length of $1 \mu \mathrm{m}$, which is about 1000 time steps. For $2.5 \times 10^{8}$ particles, and 1000 operations/timestep/ particle, a computation requires $2.5 \times 10^{11}$ operations/timestep. At 8 MFlops, this means that a timestep takes about 40 seconds. Computing the time it takes a detonation to travel $1 \mu \mathrm{m}$ then requires 40,000 seconds, which is about 11 hours. The problem, then, is finding a realistic physical system with such small detonation cells. This, in turn, means very fast energy release.

Table 3. Current Modeling Limitations

Computer:

Physical-System Size:

Number of Particles:

Computational Timestep:

Computational Time:
CM-5E, 32 Gbytes, 8 Gflops

$0.5 \times 0.5 \times 1.0 \mu \mathrm{m}^{3}$

(Detonation cell length, $1.0 \mu \mathrm{m}$ )

$2.5 \times 10^{8}$

$40 \mathrm{~s} /$ timestep

11 hours per detonation cell length

\section{CONCLUSIONS}

The conclusion from the arguments given above is that even using the classical, model interaction potentials, we cannot easily or directly use molecular dynamics methods to simulate a detonation in nitromethane on current computers for realistic macroscopic sizes and times. Even with a teraflop computer and 100 Gbytes of memory, we could obtain at most a factor of 25 in speed, and a factor of three in particle number over what is now possible. Current rates of improvement in computers indicate that there is about a factor of two increase in speed every two years. Assuming that accessable memory is not a problem, in 10 to 15 years we could perform the computation in a reasonable amount of computer time. We have not yet begun to address the problem of how to deal with the memory and corresponding bandwith problems. These might be answered in 25-30 years. This general conclusion lead to three observations about the applicability of molecular dynamics to energetic materials research.

First, we can continue to do as we have: model scaled systems that represent faster conversion of reactants to products and faster energy release than is correct for actual physical systems. This allows us to examine the qualitative features of interactions potentials, energy transfer, crystal structure, and wave initiation and propagation. This generic approach has been successful for other methods in the past. For example, in the 1960's when the DSMC method was first developed, there were no computers large enough to compute the behavior with realistic scales of physical input data. Today, thirty years later, actual computations are possible and these are regularly compared to experiments. DSMC has entered a stage where it is a practical, useful computational tool for both basic and applied research. This might be the case in 30 years for molecular dynamics of energetic materials.

Second, we could try to find some systems for which direct simulation is possible and meaningful, for which experiments can be done. This would mean working with an explosive with a very fast energy release. Experiments on such a system may not be possible, and if they were, would be very unsafe because the material would be dangerously unstable. The very factors that would make the physical system computable today make it dangerous. 
There is a final conclusion that has to be considered very seriously. That is to consider mesoscopic scale particle dynamics in which a particle now represents a cluster of say, C-N's, in a particular configuration. Particles could, for example, represent pieces of crystals that are on the scale of microns. Then we would not be attempting to represent the microscopic scale deterministically from first principles, as we are doing now with molecular dynamics. Instead, we would be attempting to describe a mesoscopic structure. This is a more phenomenological approach that could bridge the current gap between microscopic information and macroscopic behavior. A current argument in favor of this approach is that we do not even properly represent the microscopic behavior in our molecular dynamics computations now. The potentials are not quite correct: we are not quite correctly representing a crystal lattice, and we do not include the full chemical reactions. In some ways, our input data is as crude as the crudest Navier-Stokes computation of a detonation.

In summary, we believe that application of molecular dynamics to simulate the behavior of energetic crystals is truly a grand challenge in computing. We do not believe that we can perform viable direct simulations of such systems, given current computers, for the next 25-30 years. Many of the Grand Challenge problems that have driven the development of modern computing capabilities were in the same situation 20 years ago, and it is that international challenge that has brought us where we are today.

\section{ACKNOWLEDGMENTS}

This work is based on observations made during the authors' many years of working with Simone Odiot, who has inspired and guided our research on energetic materials. The authors would like to acknowledge the many helpful conversations with Choong $\mathrm{Oh}$, on the validity of various theoretical approaches to manybody problems, Kay Howell on projections of the future of computational resources, and Robert Sinkovits on the limits of our current computations. This work has been funded by the Office of Naval Research, the Naval Research Laboratory, and the Advanced Research Projects Agency.

\section{REFERENCES}

[1] Bird G.A., Molecular gas dynamics and the direct simulation of gas flows (Clarendon Press, Oxford, 1994).

[2] Karo A.M., Walker F.E., DeBoni T.M., and Hardy J.R., Prof. Aero. Astro 94 (1983) 405-415.

[3] Peyrard M., Odiot S., and Lavenir E., C.R. Acad. Sci. 299, Sŕie II, 917 (1984).

[4] Peyrard M., Odiot S., Lavenir E., and Schnur J.M., J. Appl. Phys. 57, 2626 (1985).

[5] Peyrard M., Odiot S., Oran E., Boris J., and Schnur J., Phys. Rev. B 33 (1986) 2350-2363.

[6] Tsai D.H. and Trevino S.F., J. Chem. Phys. 81 (1984) 5636-5637.

[7] Lambrakos S.G., Peyrard M., Oran E.S., and Boris J.P., Phys. Rev. B 39 (1989) 993-1005.

[8] Elert M.L., Deaven D.M., Brenner D.W., and White C.T., Phys. Rev. B 39 (1989) 1453-1456.

[9] Phillips L., J. Phys.: Cond. Matter 5, 473-6376 (1993). 